\title{
Impact of alerts mailed to prescribers related to patient discontinuation of antidepressant medications
}

\author{
Ronald Lyon ${ }^{1}$ \\ ${ }^{1}$ Hampton University
}

May 5, 2020

\begin{abstract}
Prior research demonstrates that improved medication adherence can produce substantial total health care savings. However, limited evidence exists to support the value of interventions to improve medication adherence. This article describes a method using alerts mailed to prescribers to improve adherence to antidepressants. The primary aim of the study evaluated the impact of written, patient-specific medication adherence alerts mailed to prescribers on overall depression-related health care costs associated with the use of antidepressant medications. The study used a clinical alert system that integrates medical and pharmacy claims data to identify and notify prescribers about patient-specific care gaps in the outpatient setting. This retrospective and observational study used health care claims from a high-risk population to match patients, identify conditions, and identify early discontinuation of antidepressant medications. The claims also allowed us to calculate changes in overall health care costs related to depression. The results show that mailing an alert to the prescriber increases the number of patients that have prescriptions for antidepressants six months after an alert from approximately $8 \%$ to $35 \%$. The analysis further indicated that when a patient restarts their antidepressant and continues for at least six months, their follow-up medical plus pharmacy costs will be approximate $\$ 2,671$ less than if they remain non-compliant. The study supports the use of a computer system to integrate health care claims data, identify care gaps, and generate written alerts to be mailed to prescribers as a means to improve patient adherence and lower total health care costs in patients receiving antidepressants.
\end{abstract}

\section{Introduction}

Research has demonstrated that improved medication adherence can produce substantial total health care savings. However, limited evidence exists to support the value of interventions to improve medication adherence. This article describes a successful method using mailed alerts to prescribers to improve adherence to antidepressant medications.

\section{Impact of adherence on overall health care costs}

A systematic review and meta-analysis indicated that improved adherence for four chronic vascular diseases could lower total health care costs (Roebuck et al., 2011). The annual savings per patient ranged from $\$ 1,258$ for dyslipidemia to $\$ 7,823$ for congestive heart failure.

Another systematic review added further support to the significant gaps that exist with medication adherence (Krueger et al., 2005). The review reported adherence rates that ranged from $17 \%$ to $80 \%$ and highlighted the barriers that lead to decreased adherence. 


\section{Limited value of interventions to improve medication adherence}

The Cochrane Consumers and Communication Group published an extensive review of interventions to improve medication adherence Nieuwlaat et al. (2014). The group concluded that the methods were complex and showed limited effectiveness. Another Cochrane Collaborative meta-analysis concluded that there is insufficient evidence for medication management services on improving medication adherence (Viswanathan et al., 2015). These reports show the need for further research on methods to improve adherence.

\section{Prescriber impact on medication adherence}

Koulayev et al published two reports that provide insight into the factors that affect a patient's decision to remain adherent (Koulayev et al., 2013) (Koulayev et al., 2016) . In particular, they found that the quality of the match between the patient and their physician had a substantial influence on the variation in patient adherence rates.

\section{Hypothesis}

A patient's prescriber may have the most influence on their medication adherence. This influence may relate to more trust and comfort with their prescriber rather than interventions delivered by providers that they have not previously met. They have personally selected their prescriber and likely have had multiple interactions with them if they have a chronic condition requiring medications. However, prescribers do not know whether a patient follows their advice and fills and remains adherent to their medications. Written notifications may provide prescribers with this insight into patients with potential adherence issues and allow them to determine their preferred approach to work with their patient.

\section{Objectives}

The primary aim of the study evaluated the impact of written, patient-specific medication adherence alerts mailed to prescribers on overall behavioral health care costs associated with the use of antidepressant medications.

The objectives consisted of the following analyses:

1. Compare the percentage of patients that have antidepressant medications six months after initial discontinuation based on whether their prescriber was mailed an alert or not.

2. Determine the difference in follow-up health care costs for patients that have antidepressant medications six months after the initial alert compared to those that did not.

3. Estimate the return on investment (ROI) based on differences in change rates, follow-up health care costs, and cost per intervention.

\section{Methods}

\section{Study design}

This retrospective and observational study used health care claims to match patients, identify conditions, and identify early discontinuation of antidepressant medications. The claims also allowed the study to calculate changes in overall health care costs related to depression. 


\section{Setting}

The analysis used claims data from July 2015 through March 2017 for patients in a high-risk population. The program enrolled patients with select chronic conditions, including those with a behavioral health condition, into a care management program. The most frequent behavioral health conditions included psychotic (e.g., depression) and mood (e.g., depression, bipolar, anxiety) disorders.

\section{Intervention}

This study used a clinical alert system that integrates medical and pharmacy claims data to identify and notify prescribers about patient-specific care gaps in the outpatient setting. The system uses over 17,000 rules to analyze the integrated claims to identify a variety of care gaps primarily related to patient adherence to recommended therapy. This study evaluates the impact of warning prescribers about potential early discontinuation of antidepressants. The system mails each prescriber an alert of the potential early discontinuation to allow them to assess the clinical significance and determine the best course of action for their patient. The alert includes a medication profile for their review. The profile limits the claims shown to

only those directly related to the care gap. The limited profile makes it easier to review and limits disclosure of sensitive conditions not directly related to the gap.

\section{Participants}

The system screened all plan enrollees with diagnoses on claims data that indicated depression.

\section{Patient exclusions}

The study excluded the following patients from the analysis:

- Those that did not remain in the program for at least twelve (12) months following the alert; and

- Those hospitalized within the ninety (90) days before the alert.

\section{Patient matching}

For the analysis of follow-up health care costs the study matched patients using:

- Patient age at 10-year intervals (e.g., 10-19 years of age);

- Gender;

- Same alert (early discontinuation of medications for depression);

- Same condition (depression, based on medical claims);

- Number of months available for follow-up following an alert; and

- Similar prior quarter total health care claims costs within $\$ 500$ ranges as an estimate of patient disease severity. The use of starting healthcare costs may the impact of regression to the mean.

\section{Matching of medical and pharmacy claims}

The system grouped medical claims using International Classification of Disease (ICD) codes grouped into higher level Clinical Classification System (CCS) codes. The Centers for Medicare and Medicaid Services uses the CCS for their analyses. 
The system used a clinical indications module from First Databank to match pharmacy claims to the pertinent medical condition grouped by CCS. The system also conducted further matches by use of other medical claims and prescriber specialty to further increase the accuracy of the match.

\section{Health care costs by condition}

The analysis of health care costs only included medical claims with indications for depression and medications with indications to treat those conditions. The indications include FDA-approved indications and off-label uses recognized in compendia such as AHFS-DI, Clinical Pharmacology, and DRUGDEX. The costs included both plan and patient paid amounts as reported on the approved claims.

\section{Operational costs}

Operational costs include costs directly related to delivering the service. It does not include indirect costs such as sales and marketing. The actual budgeting and reporting processes tracked these costs.

\section{Determination of early discontinuation}

The system identified early discontinuation of antidepressants using the following criteria:

- Patients included must have received at least 60 days of antidepressant medication before the discontinuation. This requirement increases the likelihood that the patient found the current medication acceptable.

- Mapped International Classification of Disease (ICD) diagnoses on the claims to the Clinical Classification System (CCS) conditions (i.e., depression) (CCS code 5.8.2).

- Used the First Databank Indications module to determine whether a patient has any current antidepressant used to treat depression.

- Compared the date of the last refill and quantity to determine the projected date the patient will run out of medication.

- The system flagged patients that were more than 15 days late to refill their current medication or receive a new prescription for a medication indicated for the same condition as an early discontinuation. If a patient received a prescription for a medication that was indicated for the treatment of depression, even if a switch from their original medication, then the system considers that a successful change.

\section{Change rate: changed versus unchanged early discontinuation care gaps}

The system only identified an alert as resulting in a change if the patient was receiving an antidepressant medication and adherent six months after the time of the alert generation. A patient with sufficient supply over the prior four months to provide at least $80 \%$ of a minimum daily dose was considered to be adherent. The change rate compares the number of patients on a medication indicated for their condition after six months based on whether the program mailed the prescriber an alert or not.

\section{Cost-differences}

The cost difference calculates the difference in follow-up health care costs between quarters two (2) and four (4) following an alert, for patients that received medication for their condition at six (6) months compared to those that had not. The data showed that, on average, changes to therapy occurred between the second and third month following the alert. The time to identify a change in therapy relates to the time it takes to prepare and mail the letters in addition to the time it takes for the prescriber to evaluate and intervene with their patient. Therefore, the first quarter following the alert was not used to compare differences in costs. 


\section{Statistical methods}

The statistical analyses used SOFA (Statistics Open for All) version 1.4.6.

\section{Results}

The study objectives included:

- Change rate: Compare the percentage of patients that have antidepressant medications six months after initial discontinuation based on whether the program mailed the prescriber an alert or not.

- Cost-differences: Determine the difference in follow-up health care costs for patients that have antidepressant medications six months after initial compared to those that did not.

- ROI: Estimate the return on investment (ROI) based on differences in change rates, follow-up health care costs, and cost per intervention.

\section{Change rate}

The change rate compares the percentage of patients on a medication indicated for their condition after six months based on whether the program mailed the prescriber an alert or not. There were three months where alerts were generated and not mailed for the plan. The study compared the change rates for those months against the months when the program mailed alerts.

These results were analyzed using a chi-square test. The contingency table shows the observed cell totals

$\begin{array}{ccccc} & \text { Change } & \text { No Change } & \text { Row Totals } & \text { Change Rate } \\ \text { Alerts Mailed } & 1354(1147.29) & 2501(2707.71) & 3855 & 0.35 \\ \text { Alerts not mailed } & 79(285.71) & 881(674.29) & 960 & 0.08 \\ \text { Column Totals } & 1433 & 3382 & 4815 & \end{array}$

Table 1: Change rates for antidepressants

and the (expected cell totals) for each cell. The chi-square statistic is 265.9405 . The p-value is $; .00001$. The result is significant at $\mathrm{p} ; .05$.

The results show that mailing an alert to the prescriber increases the number of patients that have prescriptions for antidepressants six months after an alert from approximately $8 \%$ to $35 \%$. This absolute increase of $27 \%$ suggests sending four alerts will result in one additional patient receiving antidepressant medication six months following an alert $(\mathrm{NNT}=4)$.

\section{Cost differences}

The cost difference calculates the difference in follow-up health care costs for the second through fourth quarters following an alert, for patients that received medication for their condition at six months following an alert compared to those that had not. The analysis eliminated outlier patients with greater than $\$ 50,000$ in follow-up costs.

The analysis indicated that when a patient restarts their antidepressant and continues for at least six months, their follow-up medical plus pharmacy costs will be approximate $\$ 2,671$ less than if they remain non-compliant (Table 2).

Thes results were analyzed using an independent t-test. 


$\begin{array}{ccccccc}\text { Group } & \mathrm{N} & \text { Mean } & 95 \% \text { Confidence Interval } & \text { Standard Deviation } & \text { Min } & \text { Max } \\ \text { Change } & 117 & 900.94 & \$ 595-\$ 1207 & 1689.58 & 12 & 10052.35 \\ \text { No Change } & 198 & 3572.34 & \$ 2452-\$ 4694 & 8042.17 & 3.82 & 46868.98\end{array}$

Table 2: Subsequent health care by change status

The $\mathrm{t}$ statistic is $-3,545$. The $\mathrm{p}$-value is $<0.0001$. The result is significant at $\mathrm{p}<0.05$. This analysis included far fewer patients than the initial change rate analysis due to the patient matching requirements. The study excluded patients if the analysis was unable to match patients by age, gender, condition, and prior three months health care costs. Table 3 describes patient demographics available from the claims data.

$\begin{array}{ccc} & \text { Change } & \text { No Change } \\ \text { Female (\%) } & 94(36.7 \%) & 162(63.3 \%) \\ \text { Average age (st.dev.) } & 47(+/-13.5) & 48(+/-12.3) \\ \text { Male (\%) } & 23(40.0 \%) & 36(60.0 \%) \\ \text { Average age (st.dev.) } & 49(+/-11.6) & 46(+/-11.9)\end{array}$

Table 3: Available patient demographics

\section{Estimate the return on investment (ROI) based on differences in change rates, follow-up health care costs, and cost per intervention.}

The ROI was calculated based on total cost differences, change rates, and actual operating costs.

1. Cost savings per changed alert: $\$ 2,671$

2. Additional change rate when mailing an alert: 26.9

3. Additional change rate (\#2) x cost savings (\#1): $\$ 719$

4. Direct cost to identify and mail an alert to a prescriber: less than $\$ 5$

5. Return on investment $(\# 3 / \# 4): \$ 719 / \$ 5=144$

The return on investment for the early discontinuation of antidepressants exceeded 144 to 1.

\section{Discussion}

This study focused solely on early discontinuation alerts for patients receiving antidepressants for depression. The results would likely vary for other chronic conditions and medications. Subsequent analyses will evaluate other conditions.

The study demonstrated that mailing alerts to prescribers increased the percentage of patients that remain persistent and adherent to their antidepressant medications six months following an alert. A variety of reasons may result in less than a 100\% change rate following alert. These may include prescribers never reviewing the alert or patients that did not follow the advice of their prescriber. Since the alerts only use claims data to identify potential problems the alert actually may not be pertinent for the patient.

The significant savings associated with a change reflects what other studies have estimated regarding the impact of improved medication adherence. An informal, post-study, sub-analysis evaluated the distribution of costs by medical or pharmacy claims for each quarter of follow-up. As expected, patients that receive new prescriptions account for the higher costs in the changed group during the first two follow-up quarters. During the final two quarters, reduced hospitalization costs generated the overall savings. 
As described in the introduction, studies of other interventions show limited evidence of overall cost savings. While individual patients in those studies likely benefitted from the interventions the cost of the intervention negated the savings. The high return on investment (ROI) in this study can be attributed to 1) a focus on medication adherence in a condition with significant negative impacts related to poor adherence and 2) the very low cost of the intervention related to the use of technology to identify and generate the alerts to the prescribers.

\section{Limitations of the study}

This study could not evaluate in detail the reasons why some patients were adherent at six months following an alert and why others were not. There may be other variables beyond what we were able to use for matching that would impact those differences. That type of research may require a formal survey of patients and prescribers to identify potential differences.

\section{Conclusion}

The study supports the use of a computer system to integrate health care claims data, identify care gaps, and generate written alerts to be mailed to prescribers as a means to improve patient adherence and lower total health care costs in patients receiving antidepressants.

Healthcare organizations should consider this type of system a supplement to other clinical programs. For example, the system could help lower acuity patients with care gaps, difficult to reach patients and high-risk patients not enrolled in a care management program.

The full system includes a wide range of alert rules that address quality indicators used in Medicare STAR ratings, NCQA standards, URAC standards, and physician incentive programs.

Further research will identify the potential savings, change rates, and return on investment for a wide variety of chronic conditions. In addition to supporting the use of written interventions, the calculations of savings associated with closing specific care gaps can be used to estimate the impact of specific interventions employed by other clinical programs.

\section{References}

Sergei Koulayev, Niels Skipper, and Emilia Simeonova. Who Is in Control? The Determinants of Patient Adherence with Medication Therapy. Technical report, oct 2013. URL https://doi.org/10.3386\% 2Fw19496.

Sergei Koulayev, Emilia Simeonova, and Niels Skipper. Can Physicians Affect Patient Adherence With Medication? Health Economics, 26(6):779-794, jun 2016. doi: 10.1002/hec.3357. URL https://doi. org/10.1002\%2Fhec. 3357 .

Kem P. Krueger, Bruce A. Berger, and Bill Felkey. Medication adherence and persistence: A comprehensive review. Advances in Therapy, 22(4):313-356, jul 2005. doi: 10.1007/bf02850081. URL https://doi.org/ $10.1007 \% 2 \mathrm{Fbf} 02850081$.

Robby Nieuwlaat, Nancy Wilczynski, Tamara Navarro, Nicholas Hobson, Rebecca Jeffery, Arun Keepanasseril, Thomas Agoritsas, Niraj Mistry, Alfonso Iorio, Susan Jack, et al. Interventions for enhancing medication adherence. The Cochrane Library, 2014.

M. C. Roebuck, J. N. Liberman, M. Gemmill-Toyama, and T. A. Brennan. Medication Adherence Leads To Lower Health Care Use And Costs Despite Increased Drug Spending. Health Affairs, 30(1):91-99, jan 2011. doi: 10.1377/hlthaff.2009.1087. URL https://doi.org/10.1377\%2Fhlthaff.2009.1087. 
Meera Viswanathan, Leila C Kahwati, Carol E Golin, Susan J Blalock, Emmanuel Coker-Schwimmer, Rachael Posey, and Kathleen N Lohr. Medication therapy management interventions in outpatient settings: a systematic review and meta-analysis. JAMA internal medicine, 175(1):76-87, 2015. 